\title{
A study of comorbidities in myasthenia gravis
}

\author{
Usha K. Misra ${ }^{1}\left[\right.$ · Jayantee Kalita ${ }^{1} \cdot$ Varun K. Singh $^{1} \cdot$ Surendra Kumar $^{1}$
}

Received: 13 December 2018 / Accepted: 19 February 2019 / Published online: 10 April 2019

(c) Belgian Neurological Society 2019

\begin{abstract}
Management of myasthenia gravis (MG) in the presence of comorbidities may be difficult. We report the effect of comorbidities in the outcome of MG. The patients with MG during 1991-2016 were included and evaluated including their demographic variables, clinical findings, Myasthenia Gravis Foundation of America (MGFA) score. The patients were categorized into early onset ( $\leq 40$ years) and late onset ( $>40$ years) MG. The comorbidities (autoimmune and miscellaneous) and iatrogenic complications were compared between early and late onset, and in good and poor outcome groups. Out of 81 patients with MG, 48 patients had early and 33 late onset. In $71(88 \%)$ patients, comorbidities were present and were autoimmune in 8 $(10 \%)$ and miscellaneous in all the patients (88\%). Iatrogenic complications were present in 54 (67\%) patients. Thymectomy was done in 19 patients; 16 had thymoma and 3 thymic hyperplasia. Myasthenic crisis occurred in 28 patients; 5 (18\%) had autoimmune and all had miscellaneous comorbidities. The patients with poor outcome had $\geq 2$ comorbidities, myasthenic crisis, leukocytosis, elevated serum bilirubin and creatinine, and increased number of hospital admissions $(P<0.05)$. Myasthenia gravis is associated with comorbidities in majority of patients especially in late onset group, and more than two comorbidities are related to poor outcome.
\end{abstract}

Keywords Myasthenia gravis · Comorbidity $\cdot$ Outcome $\cdot$ Autoimmune $\cdot$ Iatrogenic $\cdot$ Drug induced $\cdot$ Myasthenic crisis

\author{
Abbreviations \\ MG Myasthenia gravis \\ MGFA Myasthenia Gravis Foundation of America \\ MuSK Muscle specific kinase \\ LRP4 Lipoprotein-related protein 4
}

\section{Introduction}

Myasthenia gravis (MG) is an antibody-complement-mediated T-cell dependent autoimmune disorder characterized by fatigable muscle weakness associated with antibodies to acetylcholine receptor (AchR), muscle specific kinase (MuSK), lipoprotein-related protein 4 (LRP4) or agrin in the postsynaptic membrane of neuromuscular junction (NMJ) [1].

The incidence of MG is 7-23/million and prevalence is $70-320 /$ million. The mortality of MG has declined due to discovery of choline esterase inhibitors, immunomodulators,

Usha K. Misra

drukmisra@rediffmail.com; ukmisra@sgpgi.ac.in

1 Department of Neurology, Sanjay Gandhi Post Graduate Institute of Medical Sciences, Raebareily Road, Lucknow, Uttar Pradesh 226014, India thymectomy, and advancement in treatment of infections and intensive care units (ICU). As the patients with MG are living longer, a number of comorbidities have been noted. The patients with MG may be associated with autoimmune as well as non-autoimmune comorbidities along with treatment related complications which may affect the outcome. In the developing countries, there is higher frequency of infection, malnutrition, and many non-communicable diseases such as diabetes mellitus, hypertension, coronary artery disease and stroke, which are more severe and occur in younger patients. Moreover poor health infrastructure in these countries further aggravates the situation. Use of statins, and many antibiotics and antihypertensives may affect neuromuscular transmission leading to aggravation of myasthenic weakness. There is paucity of studies on the role of comorbidities in the outcome of MG, and most of the studies have evaluated the role of immunological comorbidity [2-7]. None of these studies comprehensively evaluated the role of different type of comorbidities in the outcome of MG [1, 8-11]. In the present study, we report the burden of comorbidities and their role in the outcome of the patients with MG. 


\section{Materials and methods}

The patients with MG during 2016-2018 were included in the present study. Many patients were in follow up since 1991; however, their comorbidities and complications to treatment in the last 2 years were noted. The diagnosis of MG was based on low rate repetitive nerve stimulation (RNS), acetyl choline receptor antibody or muscle specific kinase (MuSK) antibody and prostigmine test. The patients were included if two out of three tests were positive. Their demographic details, age at onset of MG, severity of MG, and systemic symptoms were noted. The duration and dose of acetyl choline esterase inhibitors (AchEI), prednisolone, azathioprine, plasmapheresis or IVIg were noted. The patients were divided into early onset ( $\leq 40$ years) and late onset ( $>40$ years) MG [12]. The severity of MG was assessed using Myasthenia Gravis Foundation of America Clinical Classification into I-V [13]. Presence of thymoma was noted. Information about thymectomy, time of surgery, and complications were noted. The presence of comorbidities was noted and divided into autoimmune and miscellaneous groups. Iatrogenic complications as well as infections and myasthenic crisis were noted.

\section{Definition of comorbidities}

\section{Autoimmune comorbidities}

The autoimmune comorbidities were those disorders which were likely to have an immune-mediated mechanism and preceded, accompanied or followed the diagnosis of MG. Presence of systemic lupus erythematosus; autoimmune thyroid disorder, rheumatoid arthritis, pernicious anemia, psoriasis, systemic vasculitis, etc., were noted.

\section{Miscellaneous comorbidities}

The miscellaneous comorbidities were those which were preceded or accompanied the diagnosis of MG and unlikely to be due to therapy of MG or have an immune-mediated mechanism.

\section{Hypertension}

History of documented hypertension, on antihypertensive therapy or blood pressure above 140/90 $\mathrm{mmHg}$ [14].

\section{Diabetes mellitus}

History of documented diabetes, on insulin or oral hypoglycemic drugs or fasting blood sugar $\geq 126 \mathrm{mg} / \mathrm{dl}$ and post-prandial blood sugar $\geq 200 \mathrm{mg} / \mathrm{dl}$ or HbA1C more than 6.5 [15]. Transient hyperglycemia during corticosteroid treatment, which returned to normal after reduction of corticosteroid was not considered as diabetes mellitus.

\section{Hyperlipidemia}

Patient on anti hyperlipidemic treatment or those with low density lipoprotein more than $100 \mathrm{mg} / \mathrm{dl}$, triglyceride $\geq 150 \mathrm{mg} / \mathrm{dl}$, and high-density lipoprotein $<40 \mathrm{mg} / \mathrm{dl}$ in men and $<50 \mathrm{mg} / \mathrm{dl}$ in women were considered to have hyperlipidemia [16].

\section{Chronic obstructive lung disease}

Cough and dyspnea with winter exacerbation for 2 years or more [17].

\section{latrogenic complications}

The iatrogenic complications were those which followed the treatment of MG and were likely to be due to a specific treatment.

Infections and myasthenic crisis during the study period were also noted.

\section{Exclusion criteria}

Unsupported MG diagnosis, incomplete data or medical follow-up of less than 6 months.

\section{Categorization of patients}

The patients with MG were categorized into MG with or without comorbidities. The patients with comorbidities were further categorized into autoimmune or miscellaneous. The patients who developed complications due to treatment of MG such as steroid induced transient hyperglycemia and weight gain, dyslipidemia, osteoporotic fracture, cataract were categorized as iatrogenic complication.

\section{Outcome}

Outcome was defined at 6 months on the basis of MGFA as good (MGFA class I and II) or poor outcome (MGFA class III-V). The number of deterioration needing hospitalization or emergency medical consultation was also noted.

\section{Statistical analysis}

The comorbidity in the patients with early onset MG ( $\leq 40$ years) was compared with late onset MG ( $>40$ years) using $X^{2}$ or Fisher exact for categorical and Student $t$ test or 
Mann-Whitney test for continuous variables. The number of hospitalization in the MG with comorbidity was compared with those without comorbidity using Mann-Whitney test. The statistical analysis was done by Statistical Package for Social Sciences (SPSS) version 18 (IBM). A variable having two tailed $P$ value of $<0.05$ was considered significant.

\section{Results}

Our results are based on 81 patients with MG, 34 (42\%) of them were females. The median age of the patients was 42 (7-75) years. At the time of presentation, 9 patients were in MGFA class 1, 23 in class II, 26 in class III, 17 in class IV and 6 in class V. Repetitive nerve stimulation test was positive in 78 (96\%) patients, AchR antibodies in 70 (86\%), antiMusk antibodies in $4(5 \%)$ and prostigmine test was positive in $78(96 \%)$ patients. Forty-eight $(59 \%)$ patients had early onset MG and 33 (41\%) late. Comorbidities were present in $71(88 \%)$ patients and included autoimmune in 8 and miscellaneous in all the 71 patients while iatrogenic complications were present in $54(67 \%)$ patients. Thymic enlargement on CT thorax was present in 27 patients and was considered thymoma in 19 and hyperplasia in 8 patients radiologically. Nineteen (23\%) patients underwent thymectomy. Histopathological examination revealed thymic hyperplasia in 3 and thymoma in 16 patients. There was no significant difference between prevalence of either of the thymic abnormality between early and late onset group. Early onset MG was more common in female than males $(P=0.002)$.

Autoimmune comorbidities were present in eight patients; autoimmune thyroiditis in two, rheumatoid arthritis in one and other autoimmune diseases including SLE in five patients. These patients had clinical symptoms and signs of respective autoantibodies. However, in another ten patients, autoantibodies were present without any clinical evidence of respective immunological disorder. These antibodies included ANA in seven, anti-ds-DNA in one, anti-thyroid peroxidase antibodies in two, and rheumatoid factor in one patient. Neither the autoimmune disorder nor the distribution of autoantibodies differs significantly between the early and late onset myasthenia gravis.

MUSK positive MG: Out of four patients with antiMUSK positive MG, none of them had autoimmune comorbidities. Among miscellaneous group, one patient had type-2 diabetes mellitus, hypertension, metabolic syndrome and coronary artery disease, one had anemia and one patient developed ischemic stroke. Regarding iatrogenic complications, one developed osteoporotic fracture.

All the patients received acetylcholine esterase inhibitors while plasmapheresis was done in $14(17 \%)$ patients, intravenous immunoglobulin was prescribed to $10(12 \%)$ patients, and mechanical ventilation to 19 (23\%) patients.
Myasthenic crisis occurred in 28 patients; 16 in early onset and 12 patients in late onset MG group.

\section{Effect of age of onset of MG on comorbidities and iatrogenic complications}

The autoimmune diseases were insignificantly commoner in early onset MG compared to late ( 3 vs $5 ; P=0.19$ ). Miscellaneous comorbidities such as diabetes mellitus ( 0 vs 9 ; $P=0.001$ ), hypertension (5 vs $11 ; P=0.01$ ), coronary artery disease ( 0 vs $4 ; P=0.04$ ) and metabolic syndrome (8 vs 16 ; $P=0.02$ ) were commoner in late onset MG (Table 1).

Iatrogenic complications such as transient hyperglycemia, drug induced weight gain, dyslipidemia, osteoporotic fractures, and cataract were similar between early and late onset MG groups.

\section{Myasthenic crisis and comorbidities}

$28(35 \%)$ patients had myasthenic crisis which was precipitated by infections in 20 , under dosing of drugs in five, offending drugs in three, drug default in one, extreme weather in two, thymectomy in two and myocardial infarction in one patient (Table 2). 19 patients needed mechanical ventilation and 22 received Bilevel Positive Airway Pressure support. Patients with comorbidities needed more frequent hospitalization (median 2, range 0-11) compared to those without comorbidities (0).

\section{Outcome}

Sixty-eight (84\%) patients had good and 13 (16\%) had had poor outcome including death in eight patients. The patients with good outcome had lower frequency of myasthenic crisis and less frequent intensive care unit admission. Patients with anti-musk antibodies and $>2$ comorbidities had significantly poorer outcome compared to fewer comorbidities (Table 3).

\section{Discussion}

In this study, comorbidities were present in $88 \%$ patients and iatrogenic complications in $67 \%$. The comorbidities were autoimmune disorders in $10 \%$ and miscellaneous in $88 \%$ patients. Amongst the miscellaneous comorbidities, diabetes, hypertension, and coronary artery disease were more common in late onset MG. Patients with comorbidities were also associated with myasthenic crisis and poor outcome. This study evaluated association of comorbidities and its influence in the outcome of MG. The patients with MG are at greater risk of autoimmune comorbidities compared to non-myasthenic population with a frequency of $25-78 \%$, especially in females and those with early 
Table 1 Distribution of comorbidities and complications in early and late onset myasthenia gravis

\begin{tabular}{|c|c|c|c|c|}
\hline & $\begin{array}{l}\text { Total number } \\
\text { of patients }\end{array}$ & Early onset $(N=48)$ & Late onset $(N=33)$ & $P$ value \\
\hline Comorbidities & $71(88 \%)$ & $41(85 \%)$ & $30(91 \%)$ & 0.46 \\
\hline (A) Autoimmune & $8(10 \%)$ & $3(6 \%)$ & $5(15 \%)$ & 0.19 \\
\hline Rheumatoid & 1 & $1 / 17$ & $0(16)$ & 0.16 \\
\hline Systemic lupus erythematosus & 5 & $1 / 29$ & $4 / 15$ & 0.26 \\
\hline Thyroid peroxidase antibodies & 2 & $1 / 16$ & $1 / 15$ & 0.58 \\
\hline (B) Miscellaneous & $71(88 \%)$ & $40(83 \%)$ & $31(94 \%)$ & 0.46 \\
\hline Diabetes & 16 & 4 & 12 & 0.002 \\
\hline Hypertension & 16 & 5 & 11 & 0.01 \\
\hline Coronary artery disease & 4 & 0 & 4 & 0.04 \\
\hline Metabolic syndrome & 24 & 8 & 16 & 0.02 \\
\hline Hypothyroidism & 2 & 1 & 1 & 0.78 \\
\hline Tuberculosis & 8 & 3 & 5 & 0.79 \\
\hline Asthma & 2 & 1 & 1 & 0.58 \\
\hline Restless leg syndrome & 2 & 0 & 2 & 0.08 \\
\hline Migraine & 2 & 1 & 1 & 0.79 \\
\hline Anemia & 15 & 10 & 5 & 0.67 \\
\hline Stroke & 2 & 1 & 1 & 0.79 \\
\hline Two or more comorbidities & $56(69 \%)$ & $30(62 \%)$ & $26(79 \%)$ & 0.12 \\
\hline Iatrogenic complications & $54(67 \%)$ & $30(63 \%)$ & $24(73 \%)$ & 0.34 \\
\hline Transient hyperglycemia & 6 & 4 & 2 & 0.70 \\
\hline Osteoporotic fracture & 2 & 1 & 1 & 0.79 \\
\hline Drug induced weight gain & 48 & 30 & 18 & 0.47 \\
\hline Dyslipidemia & 27 & 12 & 15 & 0.055 \\
\hline Cataract & 2 & 1 & 1 & 0.79 \\
\hline
\end{tabular}

SLE systemic lupus erythematous, VAP ventilator associated pneumonia, TPO thyroid peroxidase, $M U S K$ muscle specific kinase antibodies
Table 2 Various precipitating factors of myasthenic crisis

\begin{tabular}{ll}
\hline Precipitation of crisis & $\begin{array}{l}\text { Number } \\
\text { of patients } \\
(N=28 / 81)\end{array}$ \\
\hline Infections & 20 \\
Underdosing & 5 \\
Drug default & 1 \\
Thymectomy & 2 \\
Warm weather & 2 \\
Contrast toxicity & 1 \\
Cholinergic crisis & 2 \\
Myocardial infarction & 1 \\
Offending drug & 3 \\
\hline
\end{tabular}

onset MG $[18,19]$. In other studies, the frequency of second autoimmune disorder in $\mathrm{MG}$ patients ranged from 13 to $14 \%[6,7]$. Amongst the autoimmune disorders, autoimmune thyroiditis is the commonest followed by rheumatoid arthritis and systemic lupus erythematosus [18, 20]. The autoimmune comorbidities vary in different subgroups of
MG. The patients with early onset MG have much higher frequency of autoimmune comorbidities compared to late onset. Although the latter subgroup also has higher frequency of comorbidities compared to general population. Autoimmune thyroid disorder is the most common autoimmune comorbidity with $\mathrm{MG}$ and in up to $10 \%$ in early onset MG [6]. In our study, there was only one patient with autoimmune thyroiditis in early onset group and one patient with rheumatoid arthritis, which is reported in other studies as well $[6,19]$. In a study on 75 patients with $\mathrm{MG}$, autoimmune comorbidities were present in 21 (28\%) and included autoimmune thyroid disorders in $16 \%$, rheumatoid arthritis in 4\%, systemic lupus erythematosus in $2.6 \%$ and Lambert-Eaton myasthenic syndrome in $1.3 \%$. In ten patients, the diagnosis of autoimmune disorder was established before MG [3]. In our study, autoantibodies were present in 17 (21\%) patients; rheumatoid factor in 1, antinuclear antibody in 12, anti-ds-DNA in 6 and anti-TPO antibody in 4. Autoantibodies can be found in MG without clinical evidence of respective autoimmune disorder and may be marker of a later autoimmune disorder in some patients. We, however, did not do a long term follow up to 
Table 3 Effect of comorbidities on the outcome of myasthenia gravis

\begin{tabular}{llll}
\hline Parameters & Good outcome (68) & Poor outcome (13) & $P$ value \\
\hline Comorbidities & & & \\
$\quad$ Miscellaneous & $59(87 \%)$ & $12(92 \%)$ & 0.16 \\
$\quad$ Autoimmune & $5(7 \%)$ & $3(23 \%)$ & 0.06 \\
Iatrogenic complications & $44(72 \%)$ & $10(92 \%)$ & 0.53 \\
Crisis $(28)$ & $20(29 \%)$ & $8(61 \%)$ & 0.01 \\
Admission in intensive care unit & $22(32 \%)$ & $9(69 \%)$ & 0.01 \\
Anti-muscle specific kinase antibodies & $2 / 57(3 \%)$ & $2 / 10(20 \%)$ & 0.04 \\
Comorbidities & $58(85 \%)$ & $13(100 \%)$ & 0.14 \\
Comorbidities $\geq 2$ & $43(63 \%)$ & $13(100 \%)$ & 0.01 \\
Total leucocyte count/mm ${ }^{3}$ mean $\pm \mathrm{SD}$ & $3737 \pm 1730$ & $7018 \pm 5888$ & 0.003 \\
Serum creatinine $(\mathrm{mg} / \mathrm{dl})$ & $0.9 \pm 0.21$ & $1.1 \pm 0.53$ & 0.03 \\
Serum bilirubin $(\mathrm{mg} / \mathrm{dl})$ & $0.81 \pm 0.47$ & $1.2 \pm 1.14$ & 0.02 \\
Low dose pyridostigmine & $19(28 \%)$ & $8(61 \%)$ & 0.03 \\
\hline
\end{tabular}

MUSK muscle specific kinase antibodies document the significance of these autoantibodies. Ocular myasthenia has a special link with thyroid disorders [21, 22]. In our study, eight out of nine patients with ocular MG had comorbidities. Myasthenia associated with autoimmune thyroid disease has a milder clinical course [2].

Myasthenic crisis occurred in 28 patients in our study and was associated with infection in $68 \%$. The other triggers such as under dosing or missing of drugs, stress of extreme temperature and surgery were present in $35.7 \%$. In an earlier study on 64 patients with MG, 14 (22\%) had myasthenic crisis, which was attributed to infection in 6 , surgery in 5, drug withdrawal in 2 and comorbidity in 1 patient [23]. The reported frequency of myasthenic crisis is $15-20 \%$ who require mechanical ventilator [24]. A higher frequency of myasthenic crisis in the present study may be due to a referral bias of a tertiary care hospital where advance cases are referred.

Miscellaneous comorbidities are important especially in the patients with late onset MG. Not only diabetes, hypertension and pulmonary disease offer additional risk but the drugs used in their treatment may worsen MG such as antihypertensive and statins. In our study, drugs like levofloxacin, clindamycin and verapamil were found to worsen MG leading to crisis in three patients. Myasthenia gravis patients have higher frequency of diabetes mellitus and insulin is prescribed three times more than the controls [25].

Iatrogenic complications are important especially in late onset MG. Prednisolone is the first line immunosuppressive drug and results in frequent side effects such as precipitating diabetes, hypertension, weight gain, cushingoid appearance, osteoporosis, and bone fractures. However, there was no increase in bone fractures in a registry based study [26]. In our study, one MUSK positive patient had fracture following steroid use.

\section{Limitations}

This study has retrospective design; therefore, the details of complication may not have been very accurately captured. The study has been conducted in a tertiary care teaching hospital where advanced or complicated cases are referred; hence, the result of our study may not be extrapolated to MG in general. In our hospital, there are active super specialty departments of immunology, endocrinology, and cardiology, which could have affected the referral to the respective super specialty department and may account for a lower frequency of autoimmune disorders in our study. However, this study comprehensively evaluates the effects of comorbidities in MG. Comorbidities in MG are common, and attention should be paid in managing these comorbidities with appropriate safe drugs for better outcome.

Acknowledgements We thank Mr. Shakti Kumar for secretarial help.

Author contributions UKM: Writing and drafting of manuscript. JK: Writing and drafting of manuscript. VKS: Data collection and analysis of data. SK: Data collection, analysis of data and statistical analysis.

Funding None.

\section{Compliance with ethical standards}

Ethical approval This study was approved by Institutional Ethics Committee, SGPGIMS, Lucknow INDIA. All the patients or care givers gave their written informed consent for research, which was conducted in accordance with the Helsinki Declaration.

Conflict of interest The authors declare that the have no conflict of interest. 


\section{References}

1. Gilhus NE, Verschuuren JJ (2015) Myasthenia gravis: subgroup classification and therapeutic strategies. Lancet Neurol 14:1023-1036

2. Nacu A, Andersen JB, Lisnic V, Owe JF, Gilhus NE (2015) Complicating autoimmune diseases in myasthenia gravis: a review. Autoimmunity 48:362-368

3. Tamer S, Gokce Gunes HN, Gokcal E, Yoldas TK (2016) Coexistence of autoimmune disease and autoantibodies in patients with myasthenia gravis. Neurol India 64:45-49

4. Thorlacius S, Aarli JA, Riise T, Matre R, Johnsen HJ (1989) Associated disorders in myasthenia gravis: autoimmune diseases and their relation to thymectomy. Acta Neurol Scand 80:290-295

5. Christensen PB, Jensen TS, Tsiropoulos I et al Sørensen T, Kjaer M, Højer-Pedersen E, Rasmussen MJ, Lehfeldt E (1995) Associated autoimmune diseases in myasthenia gravis. A populationbased study. Acta Neurol Scand 91:192-195

6. Mao ZF, Yang LX, Mo XA, Qin C, Lai YR, He NY, Li T, Hackett ML (2011) Frequency of autoimmune diseases in myasthenia gravis: a systematic review. Int J Neurosci 121:121-129

7. Ramanujam R, Piehl F, Pirskanen R, Gregersen PK, Hammarstrom L (2011) Concomitant autoimmunity in myasthenia gravis-lack of association with IgA deficiency. J Neuroimmunol 236:118-122

8. Gihus NE, Nacu A, Anderson JB, Owe JF (2011) Myasthenia gravis and risk of comorbidity. Eur J Neurol 22:17-23

9. Téllez-Zenteno JF, Cardenas G, Estañol B, Garcia-Ramos G, Weder-Cisneros N (2004) Associated conditions in myasthenia gravis: response to thymectomy. Eur J Neurol 11:767-773

10. Guglin M, Campellone JV, Heintz K, Parrillo JE (2003) Cardiac disease in myasthenia gravis: a literature review. J Clin Neuromuscul Dis 4:199-203

11. Johannessen K, Mygland A, Gilhus NE, Aarli J, Vik- Mo H (1992) Left ventricular function in myasthenia gravis. Am J Cardiol 69:129-132

12. Meriggioli MN, Sanders DB (2009) Autoimmune myasthenia gravis: emerging clinical and biological heterogeneity. Lancet Neurol 8:475-490

13. Jaretzki A 3rd, Barohn RJ, Ernstoff RM, Kaminski HJ, Keesey JC, Penn AS, Sanders DB (2000) Myasthenia gravis: recommendations for clinical research standards. Task Force of the Medical Scientific Advisory Board of the Myasthenia Gravis Foundation of America. Neurology 55:16-23

14. James PA, Oparil S, Carter BL, Cushman WC, Dennison-Himmelfarb C, Handler J, Lackland DT, LeFevre ML et al (2014) Evidence-based guideline for the management of high blood pressure in adults: report from the panel members appointed to the Eighth Joint National Committee (JNC-8). JAMA 311:507-520

15. American Diabetes Association (2016) Erratum. Classification and diagnosis of diabetes. Section 2. In Standards of Medical Care in Diabetes-2016. Diabetes Care 39(Suppl. 1):S13-S22. Diabetes Care. 39:1653 2016

16. Jellinger PS, Handelsman Y, Rosenblit PD, Bloomgarden ZT, Fonseca VA, Garber AJ, Grunberger G, Guerin CK et al (2017) American Association of Clinical Endocrinologists and American College of Endocrinology guidelines for the management of dyslipidemia and prevention of cardiovascular disease. Endocr Pract 23:479-497

17. Clinical history and meeting the GOLD criteria for COPD (From the Global Strategy for the Diagnosis, Management and Prevention of COPD, Global Initiative for Chronic Obstructive Lung Disease (GOLD). 2014

18. Sardu C, Cocco E, Mereu A, Massa R, Cuccu A, Marrosu MG, Contu P (2012) Population based study of 12 autoimmune diseases in Sardinia, Italy: prevalence and comorbidity. PLoS One 7:e32487

19. Fang F, Sveinsson O, Thormar G, Granqvist M, Askling J, Lundberg IE, Ye W, Hammarström L et al (2015) The autoimmune spectrum of myasthenia gravis: a Swedish population-based study. J Intern Med 277:594-604

20. Jallouli M, Saadoun D, Eymard B, Leroux G, Haroche J, Le Thi Huong D, De Gennes C, Chapelon C et al (2012) The association of systemic lupus erythematosus and myasthenia gravis: a series of 17 cases, with a special focus on hydroxychloroquine use and a review of the literature. J Neurol 259:1290-1297

21. Kaminski HJ, Li Z, Richmonds C, Ruff RL, Kusner L (2003) Susceptibility of ocular tissues to autoimmune diseases. Ann N Y Acad Sci 998:362-374

22. Chen YL, Yeh JH, Chiu HC (2013) Clinical features of myasthenia gravis patients with autoimmune thyroid disease in Taiwan. Acta Neurol Scand 127:170-174

23. Kalita J, Kohat AK, Misra UK (2014) Predictors of outcome of myasthenic crisis. Neurol Sci 35:1109-1114

24. Fink ME (1993) Treatment of the critically ill patient with myasthenia gravis. In: Ropper AH (ed) Neurological and neurosurgical intensive care, 3rd edn. Raven Press, New York, pp 351-362

25. Andersen JA, Owe JF, Engeland A, Gilhus NE (2014) Total drug treatment and comorbidity in myasthenia gravis; a populationbase cohort study. Eur J Neurol 21:948-995

26. Pouwels S, Javald M, Boer AD, de Vries F (2011) Risk of fracture in patients with myasthenia gravis: the general practice research database. Bone 48:270

Publisher's Note Springer Nature remains neutral with regard to jurisdictional claims in published maps and institutional affiliations. 